\title{
Search for a neutral Higgs boson in MSSM
}

\section{Javier Cuevas*}

Universidad de Oviedo, Spain

E-mail: Javier.Cuevas@cern.ch

The four LEP collaborations have searched for neutral Higgs bosons predicted within the MSSM. The experimental results included the searches for neutral Higgs bosons at LEP1 and LEP2 in final states as expected in the MSSM. The combined LEP data show no significant excess of events over the expectation from the Standard Model predictions. The search results are interpreted within the MSSM for a number of models in CP-conserving scenarios which lead to large exclusions in the theoretical MSSM parameter space

International Europhysics Conference on High Energy Physics

July 21st - 27th 2005

Lisboa, Portugal

* Speaker. 


\section{Introuction}

One of the more important questions in particle physics is the origin of mass and the electroweak symmetry breaking, that are believed to be generated by the Higgs mechanism. The Standard Model requires one Higgs field doublet and predicts a single neutral Higgs boson of unknown mass. Extensive searches at LEP has established a lower bound [1] of $114.4 \mathrm{GeV} / c^{2}$. The MSSM model is the SUSY extension with minimal new particle content. It has an extended Higgs sector with two doublets of Higgs fields. Two important parameters in this sector are the Higgs doublet mixing angle $\alpha$, and the ratio of the doublet vacuum expectation values $\tan \beta$. The two-doublets of Higgs fields lead to five physical Higgs bosons, of which three are neutral. In CP-conserving MSSM models, which is the case of the scenarios considered here, two of the three neutral Higgs bosons, denoted $\mathrm{h}$, for the lighter one, and $\mathrm{H}$, are $\mathrm{CP}-$ even. The third one is a $\mathrm{CP}$-odd pseudoscalar, denoted A. In $\mathrm{e}^{+} \mathrm{e}^{-}$collisions, the dominant production mechanism for the CP-even scalars is the s-channel process and the CP-odd pseudo-scalar is produced in association with either of the CP-even scalars, charged Higgs bosons, $\mathrm{H}^{+}$and $\mathrm{H}^{-}$, are also produced in pairs through a similar diagram. In most of the MSSM parameter space, only hZ and hA productions are kinematically possible at LEP energies. These processes have complementary cross-sections since the hZZ and hAZ couplings are proportional to $\sin (\alpha-\beta)$ and $\cos (\alpha-\beta)$, respectively. If kinematically allowed, hZ production dominates at low $\tan \beta$ or at large $m_{\mathrm{A}}$, while in the rest of the parameter space, it is suppressed with respect to hA pair-production. In the range of masses accessible at LEP and in most of the MSSM parameter space of the scenarios considered here, the main decays of the three neutral Higgs bosons are into the pair of heaviest fermions kinematically permitted. These different decay channels define the topologies that were searched for to cover the MSSM parameter region kinematically accessible at LEP energies.

\section{The MSSM framework}

At tree level, the production cross-sections and the Higgs branching fractions in the MSSM depend on two free parameters, $\tan \beta$ and one Higgs boson mass, or, alternatively, two Higgs boson masses, e.g. $m_{\mathrm{A}}$ and $m_{\mathrm{h}}$. Radiative corrections introduce additional parameters related to supersymmetry breaking. The parameters beyond tree level are: the top quark mass, the Higgs mixing parameter, $\mu$, the common sfermion mass term at the EW scale, $M_{\text {susy }}$, the $\mathrm{SU}(2)$ gaugino mass term at the EW scale, $M_{2}$, the gluino mass, $m_{\tilde{g}}$, and the common squark trilinear coupling at the EW scale, $A$. The radiative corrections affect the relationships between the masses of the Higgs bosons, with the largest contributions arising from the top/stop loops. As an example, the $h$ boson mass, which is below that of the $\mathrm{Z}$ boson at tree level, increases by a few tens of $\mathrm{GeV} / c^{2}$ in some regions of the MSSM parameter space due to radiative corrections. The combined LEP data are compared to the predictions of a number of MSSM "benchmark" models [2]. Within each of these noodels, the two tree-level parameters, $\tan \beta$ and $m_{\mathrm{A}}$ in the case $\mathrm{CP}$ invariance is assumed are scanned over broad ranges while the other parameters are set to fixed values.

\subsection{The CP-conserving benchmark scenarios}

Eight benchmark scenarios are considered, as suggested in Ref. [2]. The first three scenarios 
are those usually studied at LEP. They have been proposed to test the sensitivity of LEP to Higgs bosons with either masses close to the kinematical limit or decays difficult to detect. Similarly, the five other scenarios are aimed at testing the sensitivity of the Higgs boson searches at hadron colliders. The first two scenarios, called the $m_{\mathrm{h}}^{\max }$ scenario and the no-mixing scenario, differ only by the value of $X_{t}=A-\mu \cot \beta$, the parameter which has the largest impact on the mass of the $\mathrm{h}$ boson. The $m_{\mathrm{h}}^{\max }$ scenario leads to the maximum possible $\mathrm{h}$ mass as a function of $\tan \beta$. The no-mixing scenario is its counterpart with vanishing mixing. These two scenarios are quite representative of the sensitivity of LEP. The third scenario, called the large $\mu$ scenario, has a large and positive value of $\mu$ and a relatively small value of $m_{\tilde{g}}$. It predicts at least one scalar Higgs boson with a mass within kinematic reach at LEP2 in each point of the MSSM parameter space. This scenario was designed to test the sensitivity of LEP through analyses that could not benefit from the b-tagging capabilities of the experiments. Among the five other benchmark scenarios, three are variants of the $m_{\mathrm{h}}^{\max }$ and no-mixing scenarios. The changes in the Higgs boson mass spectrum and properties are small. The last two scenarios have been proposed to test potential difficult cases for the searches at hadron colliders. Hence, the gluophobic scenario presents regions where the main production channel at the LHC, gluon fusion, is suppressed. In the small $\alpha$ scenario, important decay channels at the Tevatron and at the LHC, $\mathrm{h} \rightarrow \mathrm{b} \bar{b}$ and $\mathrm{h} \rightarrow \tau^{+} \tau^{-}$, are suppressed when $\alpha$ is small, which occurs at large $\tan \beta$ and moderate $m_{\mathrm{A}}$ with the chosen set of parameter values.

\section{Comparisons of the data with the expected background}

The distribution of $1-\mathrm{CL}_{\mathrm{b}}$ provides a convenient way of studying the agreement between the data and the expected background and of discussing of any local excess in the data. Figure 1 shows the distribution of this variable determined from the LEP combined searches for the CP-conserving benchmark $m_{\mathrm{h}}^{\max }$ scenario. Over the largest part of the parameter space the local excesses are smaller than two standard deviations. In the $m_{\mathrm{h}}^{\max }$, the lowest value of $1-\mathrm{CL}_{\mathrm{b}}=1.3 \times 10^{-3}$ lies within the band at $m_{h}$ around $100 \mathrm{GeV} / c^{2}$ and corresponds to 2.5 standard deviations.
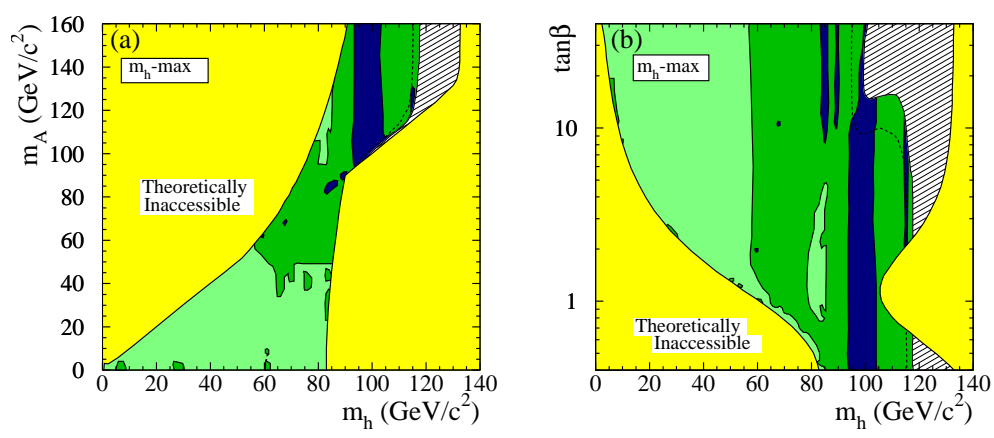

Figure 1: Contours of observed 1-CL $\mathrm{CL}_{\mathrm{b}}$ values indicating the statistical significances of local excesses in the data. In the medium-grey regions the data deviate by less than one standard deviation from the expected background, while in drak-grey regions the deviation is between two and three standard deviations. 


\section{Results interpreted in the CP-conserving MSSM scenario}

The exclusion limits are obtained from the values of $\mathrm{CL}_{\mathrm{s}}$, and for an assumed top quark mass of $m_{t}=174.3 \mathrm{GeV} / c^{2}$. For the benchmark $m_{\mathrm{h}}^{\max }$ scenario the exclusions are shown in Figure 2. Values of $m_{\mathrm{h}}$ and $m_{\mathrm{A}}$ less than $92.8 \mathrm{GeV} / c^{2}$ and $93.4 \mathrm{GeV} / c^{2}$, are excluded at $95 \%$ c.l. and values of $\tan \beta$ between 0.7 and 2.0 are excluded but this range varies considerably with $m_{t}$ and also with $M_{S U S Y}$. Similar exclusion limits have been obtained in the other benchmark scenarios studied. The combined LEP data do not reveal any excess of events which would indicate the production of Higgs bosons within the MSSM model. It has been shown that in all the scenarios presented the searches performed at LEP have excluded sizeable domains of the theoretically allowed parameter space.
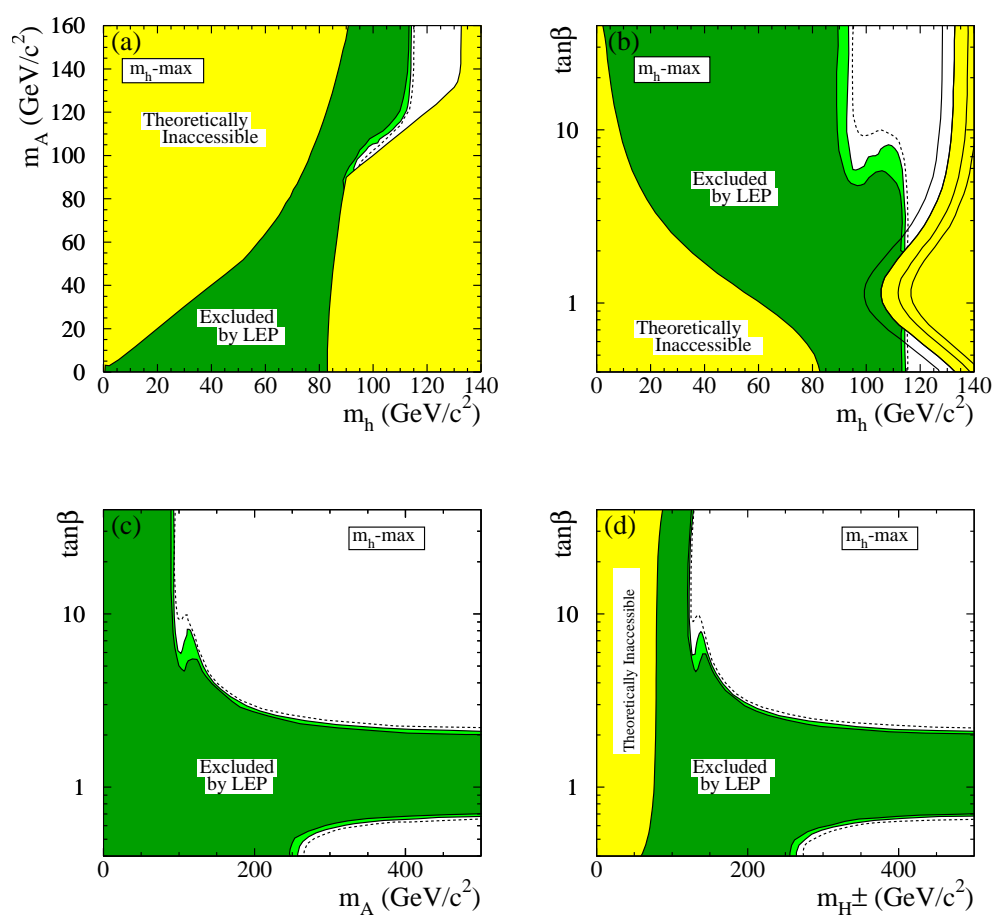

Figure 2: The MSSM exclusions at $95 \%$ c.l. for the $m_{\mathrm{h}}^{\max }$ benchmark scenario with $m_{t}=174.3 \mathrm{GeV} / c^{2}$ The dashed lines indicates the boundaries of the region s expected to be excluded at $95 \%$ c.l. on the basis of Monte Carlo simulations with no signal. In the $\left(m_{\mathrm{h}}, \tan \beta\right)$ projection, the upper boundary of the parameter space is indicated for four top quark masses.

\section{References}

[1] ALEPH, DELPHI, L3, OPAL Collaborations and the LEP Higgs working group, Phys. Lett. B565 (2003) 61.

[2] M. Carena, S. Heinemeyer, C. Wagner and G. Weiglein, CERN-TH/99-374

M. Carena, S. Heinemeyer, C. Wagner and G. Weiglein, Eur. Phys. J. C26 (2003) 601. 